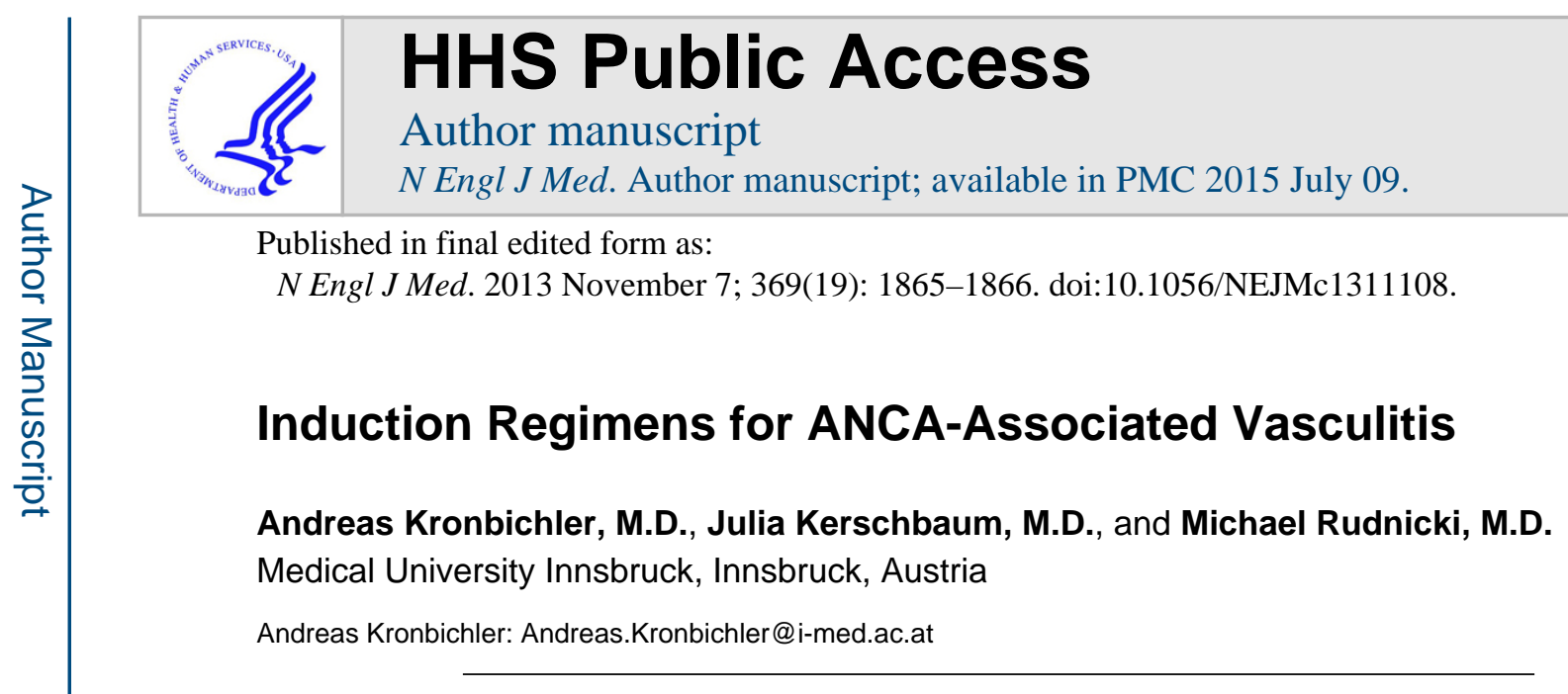

\title{
TO THE EDITOR
}

In the 18-month follow-up report of the Rituximab in ANCA-Associated Vasculitis (RAVE) trial, Specks et al. (Aug. 1 issue) ${ }^{1}$ reported a noninferiority of intravenous rituximab (375 mg per square meter of body-surface area administered weekly for 4 weeks) as compared with oral cyclophosphamide (taken daily) followed by azathioprine. Complete remission was maintained in 39\% of the patients in the rituximab group and in 33\% of the patients in the comparison group. As compared with the results of the CYCLOPS trial, the relapse rate in the control group exceeded expectations (20.8\% in the CYCLOPS trial vs. $29 \%$ in the RAVE trial), although the median follow-up in the CYCLOPS trial was 4.3 years. ${ }^{1,2}$ One explanation might be the rigorous tapering of glucocorticoids in the RAVE trial, because early discontinuation of glucocorticoids is one of the most significant risk factors for relapse. ${ }^{3}$ These differences were not discussed thoroughly, but clinical trials should address the need to prevent relapses in order to reduce treatment-related adverse events.

Repeated administration of rituximab has shown encouraging results. ${ }^{4}$ Further trials that are now being conducted will reassess these preliminary data. Nonetheless, the results of the RAVE trial have disproved the hypothesis that prolonged immunosuppressive treatment is absolutely necessary in antineutrophil cytoplasmic antibody (ANCA)-associated vasculitis.

\section{References}

1. Specks U, Merkel PA, Seo P, et al. Efficacy of remission-induction regimens for ANCA-associated vasculitis. N Engl J Med. 2013; 369:417-27. [PubMed: 23902481]

2. Harper L, Morgan MD, Walsh M, et al. Pulse versus daily oral cyclophosphamide for induction of remission in ANCA-associated vasculitis: long-term follow-up. Ann Rheum Dis. 2012; 71:955-60. [PubMed: 22128076]

3. Walsh M, Merkel PA, Mahr AD, Jayne D. Effects of duration of glucocorticoid therapy on relapse rate in antineutrophil cytoplasmic antibody-associated vasculitis: a meta-analysis. Arthritis Care Res (Hoboken). 2010; 62:1166-73. [PubMed: 20235186]

4. Smith RM, Jones RB, Guerry MJ, et al. Rituximab for remission maintenance in relapsing antineutrophil cytoplasmic antibody-associated vasculitis. Arthritis Rheum. 2012; 64:3760-9. [PubMed: 22729997]

\footnotetext{
No potential conflict of interest relevant to this letter was reported.
} 